\title{
Knockdown of NANOG enhances chemosensitivity of liver cancer cells to doxorubicin by reducing MDR1 expression
}

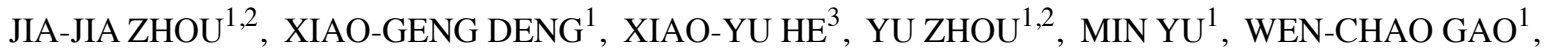 \\ BING ZENG $^{1}$, QUAN-BO ZHOU ${ }^{1,2}, \mathrm{ZHI}_{-\mathrm{HUA} \mathrm{LI}^{2} \text { and RU-FU CHEN }}{ }^{1,2}$ \\ Departments of ${ }^{1}$ Surgery and ${ }^{2}$ Oncology, Sun Yat-sen Memorial Hospital, \\ Sun Yat-sen University; ${ }^{3}$ Laboratory of Biomechanics and Physiology, \\ Guangdong Provincial Institute of Sports Science, Guangzhou, Guangdong, P.R. China
}

Received January 5, 2014; Accepted March 4, 2014

DOI: 10.3892/ijo.2014.2347

\begin{abstract}
Multidrug resistance (MDR) is one of the major reasons for the failure of liver cancer chemotherapy, and its suppression may increase the efficacy of chemotherapy. NANOG plays a key role in the regulation of embryonic stem cell self-renewal and pluripotency. Recent studies reported that NANOG was abnormally expressed in several types of tumors, indicating that NANOG is related to tumor development. However, the correlation between NANOG and liver cancer chemoresistance remains uncertain. In this study, RNA interfere technology was employed to knock down NANOG expression in HepG2 human liver cancer cells. We found that the knockdown of NANOG expression in NANOG siRNA-transfected HepG2 cells resulted in decreased colony formation rate and cell migration compared to control HepG2 cells. In addition, HepG 2 cells were treated with doxorubicin to evaluate the chemosensitivity to doxorubicin. We found that the doxorubicin sensitivity of HepG2 cells was increased with downregulation of NANOG expression. The expression of MDR1 at both mRNA and protein levels was decreased in HepG2 cells when NANOG was knocked down. These findings suggest that the knockdown of NANOG in HepG2 human cells resulted in decreased MDR1 expression and increased doxorubicin sensitivity, and NANOG could be used as a novel potential therapeutic target to reverse multidrug resistance of liver cancer.
\end{abstract}

\section{Introduction}

Hepatocellular carcinoma (HCC) is the third most frequent cause of cancer-related death $(1,2)$. In patients with HCC, the best treatment is radical operation of the tumor. However, only

Correspondence to: Professor Ru-Fu Chen, Department of Surgery, Sun Yat-sen Memorial Hospital, Sun Yat-sen University, 107 West Yan Jiang Road, Guangzhou, Guangdong 510120, P.R. China E-mail: sums1866@126.com

Key words: hepatocellular carcinoma, NANOG, doxorubicin, multidrug resistance gene 1 , chemosensitivity a small proportion of HCC patients can undergo a radical operation, and even in patients who are suitable for radical surgery, the risk of recurrence is high. Thus, chemotherapy is an important alternative therapeutic strategy for most HCC patients (3). However, chemotherapy in many HCC patients is often ineffective due to multidrug resistance (MDR) that cancer cells can developed against a variety of structurally and functionally diverse chemotherapeutic agents (4). Many studies have indicated that alterations in target gene expression are correlated with MDR, one form of MDR is caused by overexpression of P-glycoprotein, an MDR1 gene product (5). P-glycoprotein is a transmembrane phosphoglycoprotein belonging to the ATP-binding cassette (ABC) superfamily, which pumps anticancer agents out of the cells leading to reduced intracellular drug concentration and cytotoxicity (6). Inhibition of MDR1 gene or P-glycoprotein in malignant cancer cells can restore their sensitivity to anticancer agents.

The NANOG gene, a member of the homeobox family of DNA binding transcription factors, was recently identified as a master molecule essential for maintaining self-renewal and pluripotency of embryonic stem cells (ESCs) $(7,8)$. Recent accumulating evidence showed that abnormal expression of NANOG is detected in several types of human cancers, such as embryonic carcinoma (9), breast cancer (10), prostate cancer (11), glioma (12), retinoblastoma (13) and colon cancer (14). Downregulation of NANOG inhibits tumor cells development associated with an inhibition of cell proliferation, clonal expansion and clonogenic growth of tumor cells, indicating that NANOG expression in human cancer cells is biologically functional in regulating tumor development (15). In addition, it has been reported that overexpression of NANOG may induce chemoresistance to cisplatin in prostate and breast cancer cells (16) and NANOG siRNA plus cisplatin may enhance the sensitivity of chemotherapy in esophageal cancer (17), suggesting that NANOG may have a potential role in MDR. Comprehensive and systematic studies of NANOG expression in human tumor cells have proceeded, however, research of the correlation between NANOG expression and liver cancer cell multidrug resistance is lacking. The molecular mechanisms of NANOG in regulating liver cancer cell multidrug-resistance needs clarification. 
Table I. Primer sequences used in real-time RT-PCR.

\begin{tabular}{llll}
\hline Gene & Accession no. & \multicolumn{1}{c}{ Forward primer } & \multicolumn{1}{c}{ Reverse primer } \\
\hline NANOG & NM_024865.2 & 5'-CTCTCCTCTTCCTTCCTCCAT-3' & 5'-TTGCGACACTCTTCTCTGC-3' \\
MDR1/ABCB1 & NM_000927.4 & 5'-CTTCAGGGTTTCACATTTGGC-3' & 5'-GGTAGTCAATGCTCCAGTGG-3' \\
$\beta$-actin & NM_001101 & 5'-CGGCATCGTCACCAACTG-3' & 5'-GGCACACGCAGCTCATTG-3' \\
\hline
\end{tabular}

In the present study, in order to determine whether NANOG plays an important role in human liver cancer MDR, we used RNA interfere technology to silence NANOG mRNA and to examine the effect of NANOG on the biological characteristics including drug resistance of doxorubicin in human HepG2 liver cancer cells. We demonstrated that the knockdown of NANOG resulted in decreased colony formation rate and cell migration compared to control HepG2 cells. Furthermore, the chemosensitivity of HepG2 cells to doxorubicin was increased and the expression of MDR1 gene at both mRNA and protein levels was decreased in HepG2 cells when NANOG was knocked down. These results indicate that NANOG may have a particular important role in regulating chemosensitivity of human liver cancer. Our findings provide new insight into the mechanism of NANOG regulating MDR in HCC.

\section{Materials and methods}

Cell lines and culture. The human liver carcinoma cell line HepG2 was purchased from ATCC (Manassas, VA, USA), and stored in our laboratory. Cells were maintained in RPMI-1640 medium supplemented with $10 \%$ fetal bovine serum (FBS) (Hyclone) at $37^{\circ} \mathrm{C}$ in an atmosphere of $5 \% \mathrm{CO}_{2}$ with humidity. The culture medium was changed every $24 \mathrm{~h}$.

NANOG siRNA transfection. Knockdown of NANOG expression was achieved using transfection of NANOG-siRNA. The target mRNA sequences for the NANOG-siRNA were as follows: AAC CAG ACC UGG AAC AAU UCA (GenBank accession no. NM_024865, 808-828). Non-targeting siRNA was used to control for non-specific effects. The FAM-labeled siRNAs were synthesized by Gene Chem Co., Ltd. Cells were transfected $24 \mathrm{~h}$ under standard culture conditions with $100 \mathrm{nM}$ siRNA duplexes using Lipofectamine ${ }^{\mathrm{TM}} 2000$ (Invitrogen, Carlsbad, CA, USA) following the manufacturer's protocols. The mock transfected cells were transfected with Lipofectamine $^{\mathrm{TM}} 2000$ without siRNA.

Real-time RT-PCR. Forty-eight hours after transfection, cells were harvested in TRIzol reagent (Invitrogen) and total RNA was isolated following the manufacturer's instructions. The cDNA were synthesized by using a reverse transcription kit (Takara Bio, Dalian, China), and the quantitative real-time polymerase chain reaction (PCR) was conducted with a SYBR Premix Ex Tag (Takara Bio). The PCR reaction proceeded as follows: $95^{\circ} \mathrm{C}$ for $30 \mathrm{sec}$, then 35 cycles including $90^{\circ} \mathrm{C}$ for $30 \mathrm{sec}$ and $60^{\circ} \mathrm{C}$ for $30 \mathrm{sec}$. Post-PCR melting curves confirmed the specificity of single-target amplification, and fold changes in gene expressions were normalized to housekeeping gene $\beta$-actin. The results were analyzed by LC-480 system. Gene-specific primers sets are shown in the Table I.

Western blot assay. After transfection for $48 \mathrm{~h}$, cells and supernatant of each group were collected. Proteins were extracted after break-down of cells by SDS boiling method. An equal amount of protein from whole cell lysates was separated by SDS-PAGE. Proteins were transferred onto PVDF membranes, and were blocked with 5\% non-fat milk in TBST for $1 \mathrm{~h}$ at room temperature and then incubated with antibodies against NANOG (Abcam), MDR1 (Cell Signaling Technology), and tubulin (Sigma) at $4^{\circ} \mathrm{C}$ overnight. After washing with TBST, membranes were incubated with HRP-conjugated secondary antibody (Bio-Rad) for $1 \mathrm{~h}$ at room temperature. The Supersignal ${ }^{\circledR}$ West Pico Chemiluminescent Substrate (Thermo, USA) was used to visualize protein bands on X-ray film.

Colony formation assay. The number of colonies was determined. Briefly, following transfection for $48 \mathrm{~h}$, cells were trypsinized, counted, and seeded for the colony forming assay in $60-\mathrm{mm}$ dishes at 500 cells per dish. After incubation for 14 days, colonies were stained with crystal violet and the numbers of positive cells counted. Colonies containing $>50$ cells were scored, and triplicates containing 10-150 colonies/dish were counted in each treatment.

Cell migration assay. Transwell filter migration assay is one of the most frequently used methods to analyze cell migration in vitro assays. Briefly, a total of $5 \times 10^{5}$ cells were seeded into upper chamber of the polycarbonate membrane filter inserts with $8-\mu \mathrm{m}$ pores (Corning Costar Corp., Cambridge, MA, USA) in a 12-well plate and cultured in $400 \mu \mathrm{l}$ of RPMI-1640 only medium. The lower chamber was filled with $800 \mu$ l of $10 \%$ FBS-RPMI-1640. After incubation for $48 \mathrm{~h}$, non-migrating cells in the upper chamber surface were removed with cotton swabs. Migrated cells on the bottom side of the membrane were fixed with formaldehyde for $10 \mathrm{~min}$ and stained with the three Step Stain Set kit (Richard-Allen Scientific, Kalamazoo, MI, USA). The stained membranes were cut and placed onto a glass slide, and the number of migrated cells on the bottom surface of the membrane was counted under a bright field light microscope.

Cell viability assay. Cell viability assay was performed by using a CCK8 method. Briefly, cells were seeded in 96-well plates (Corning, NY, USA). After overnight culture, HepG2 cells were transfected with NANOG siRNA or control siRNA 
A
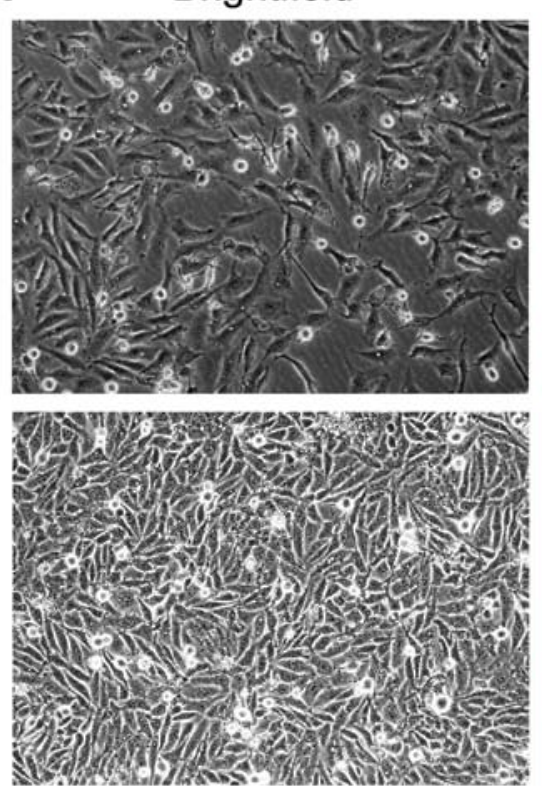

B

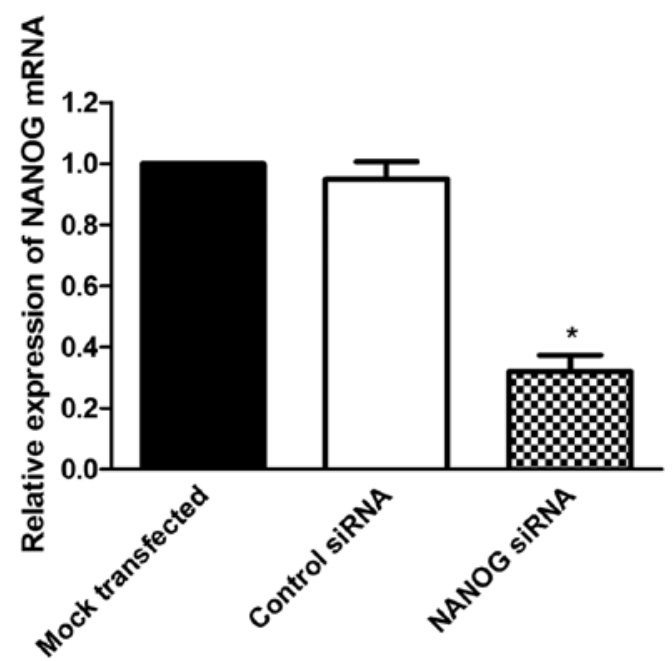

Figure 1. Knockdown of NANOG by specific siRNAs. (A) Uptake of FAM-labeled NANOG siRNA in HepG2 cells 6 or $24 \mathrm{~h}$ after transfection; (B) NANOG mRNA levels assessed by real-time RT-PCR $24 \mathrm{~h}$ after transfection in HepG2 cells treated with either NANOG siRNA, or control siRNA, or mock-transfected cells. Data were normalized by using $\beta$-actin as an internal standard. "P<0.05 vs. mock transfected cells and cntrol siRNA transfected cells. (C and D) NANOG protein levels assessed by western blot analysis $48 \mathrm{~h}$ after transfection in HepG2 cells treated with either NANOG siRNA, or control siRNA, or mock transfected cells. Tubulin was used as a loading control. ${ }^{*} \mathrm{P}<0.05$ vs. mock transfected cells and control siRNA transfected cells.

for $24 \mathrm{~h}$, then exposed to doxorubicin at final concentrations of 1 and $5 \mu \mathrm{g} / \mathrm{ml}$ for 24 or $48 \mathrm{~h}$ in a $\mathrm{CO}_{2}$ incubator, and then the viability was accessed. CCK8 assay was used to detect the chemosensitivity of cells according to the manufacturer's instructions. The absorbance at $450 \mathrm{~nm}$ was measured using a microplate reader. Six replicate wells were used for each group.

Statistical analysis. Results were presented as means of three independent experiments $( \pm$ SD). Statistical analyses were
FAM
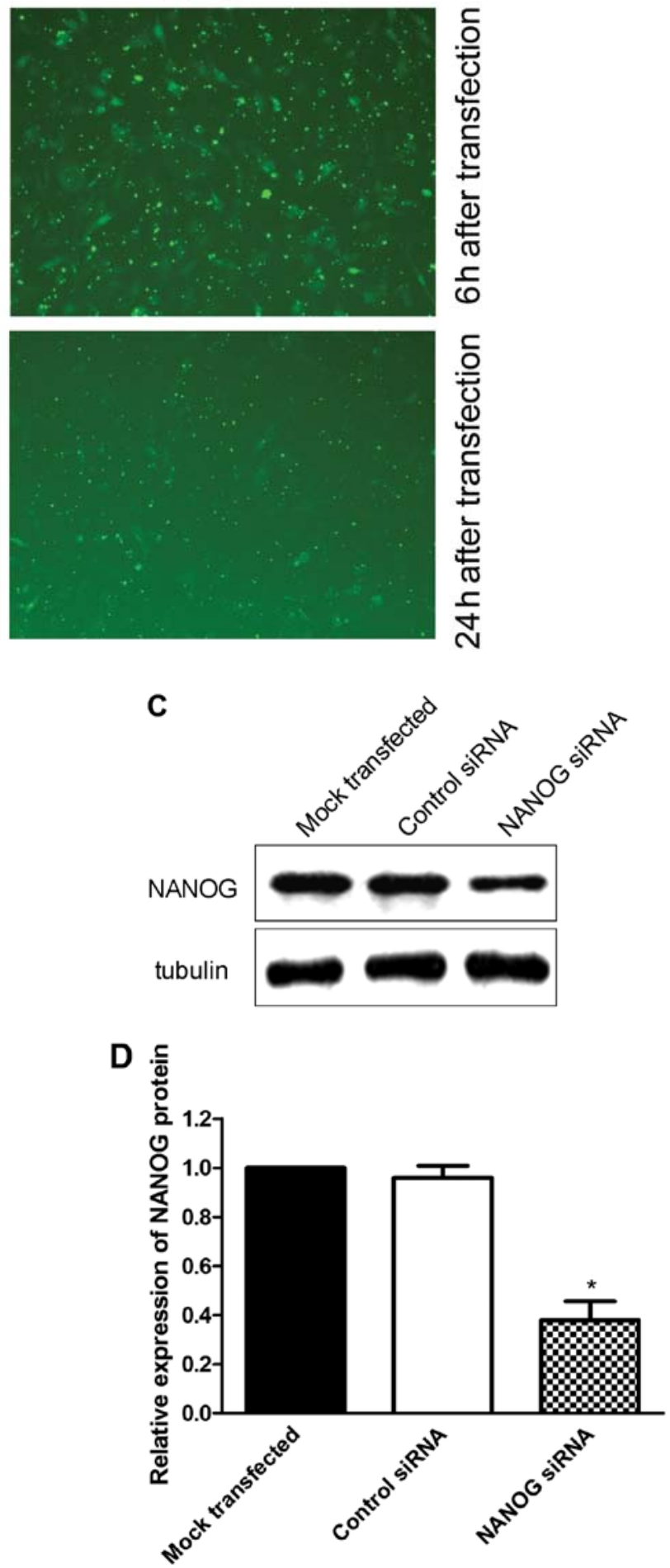

performed with the two-tailed Student's t-test or ANOVA using SPSS 13.0. $\mathrm{P}<0.05$ was considered statistically significant.

\section{Results}

Knockdown of NANOG by specific siRNA. In order to knockdown NANOG expression, the specific FAM-labeled siRNA targeting NANOG mRNA sequences was effectively transfected into the HepG2 cells by Lipofectamine ${ }^{\mathrm{TM}} 2000$. As shown in Fig. 1, transfection of HepG2 cells with NANOG 

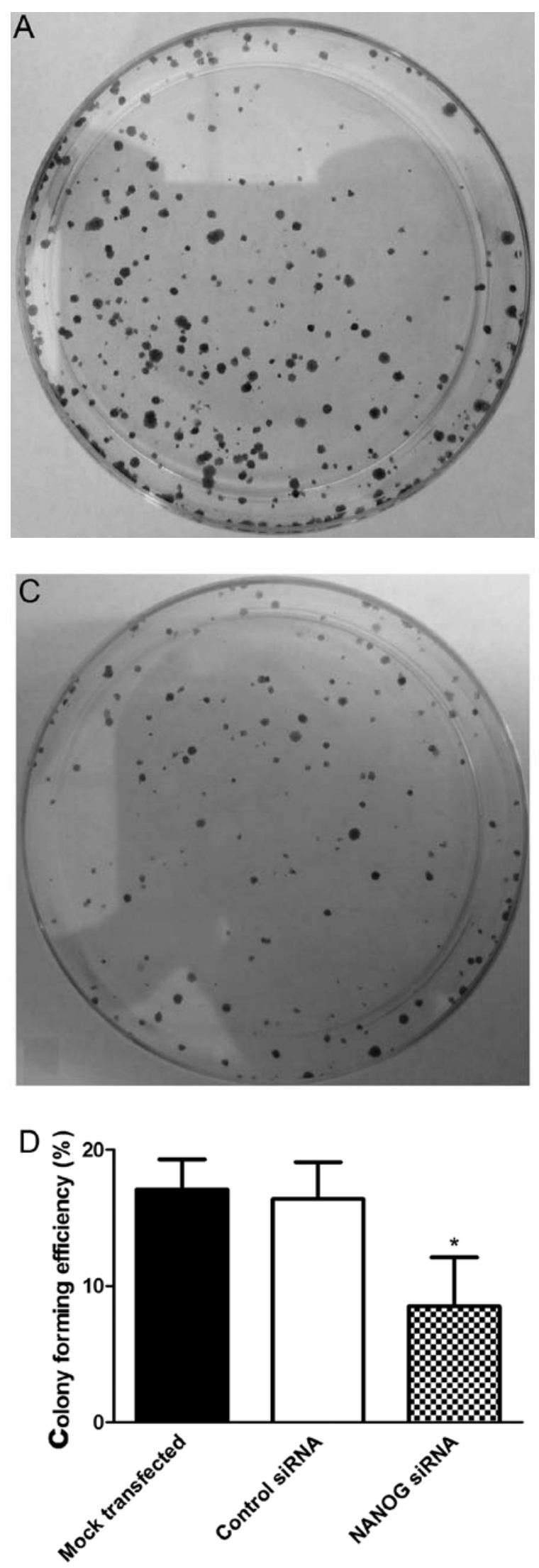

Figure 2. Knockdown of NANOG inhibited clonogenicity in HepG2 liver cancer cells. (A-C) Cell colonies of HepG2 cells transfected with NANOG siRNA were less in numbers than that of mock transfected and control siRNA tranfected cells; (A) mock transfected HepG2 cells; (B) control siRNA tranfected HepG2 cells; (C) NANOG siRNA tranfected HepG2 cells; and (D) colony formation rate. Values are mean \pm standard deviation. ${ }^{*} \mathrm{P}<0.05$ vs. mock transfected cells and control siRNA transfected cells.

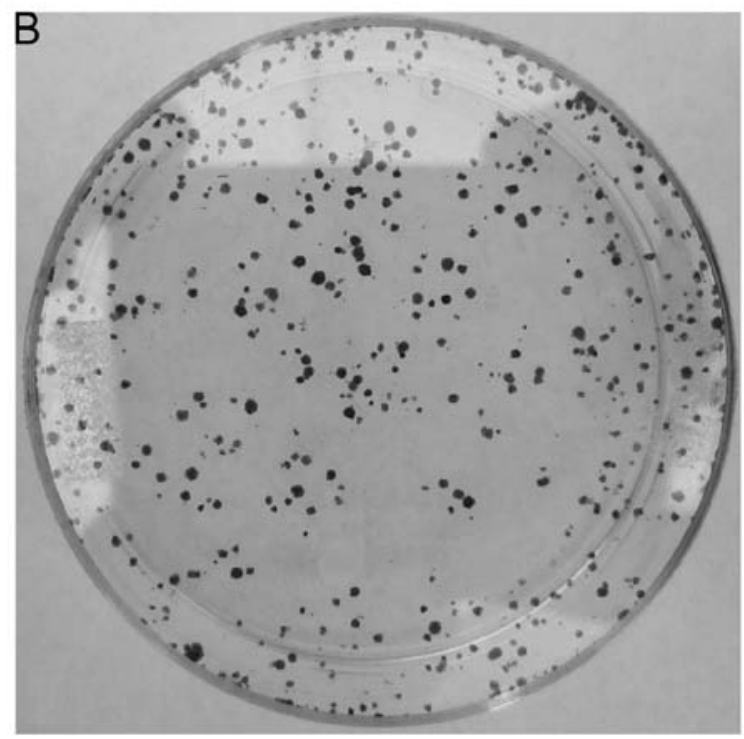

siRNA resulted in knockdown of NANOG at both the transcription and translation levels. The control siRNA transfected cells had no significant impact on NANOG expression levels compared with the mock transfected cells.

Knockdown of NANOG inhibits clonogenicity of HepG2 liver cancer cells. In order to examine the role of NANOG on the clonogenicity of HepG2 cells, we examined the effect of NANOG siRNA on cell colony formation assay. As shown in Fig. 2, clonogenicity of HepG2 cells transfected with NANOG siRNA was decreased according to the number of cell colonies, and the colony formation rate of NANOG siRNA tranfected cells was $8.5 \pm 3.6 \%$, lower than that of mock transfected and control siRNA tranfected cells $(\mathrm{P}<0.05)$.

Knockdown of NANOG inhibits cell migrating ability of HepG2 cells. The results of Transwell cell migration are presented in Fig. 3. Knockdown of NANOG expression resulted in significant inhibition of cell migration of HepG2 cells with $48.92 \pm 5.87$ cell invasion, whereas, $106.3 \pm 6.93$ and 108.1 \pm 7.45 migrated cells were observed in mock HepG2 and HepG2-s-GFP cell lines, respectively ( $\mathrm{P}<0.05$, Fig. 3).

Knockdown of NANOG sensitizes cells to doxorubicin. To evaluate the effect of NANOG on doxorubicin sensitivity of HepG2 cells, the cell viability of HepG2 cells transfected with NANOG siRNA and then exposed to doxorubicin was tested by using a CCK8 method. As shown in Fig. 4, HepG2 cells transfected with NANOG siRNA were more sensitive to doxorubicin than the mock transfected and control siRNA tranfected cells and these data indicated that the sensitivities of HepG2 to doxorubicin were enhanced by knockdown of NANOG.

Knockdown of NANOG reduced expression of MDR1 in HepG2 cells. To further evaluate the effect of NANOG on doxorubicin sensitivity of HepG2 cells, we investigated the expression of MDR1 which is regarded as an important factor on drug resistance and sensitivity of chemotherapy. As shown in Fig. 5, MDR1 expression was related closely with NANOG expression. Compared to the mock transfected HepG2 cells, 

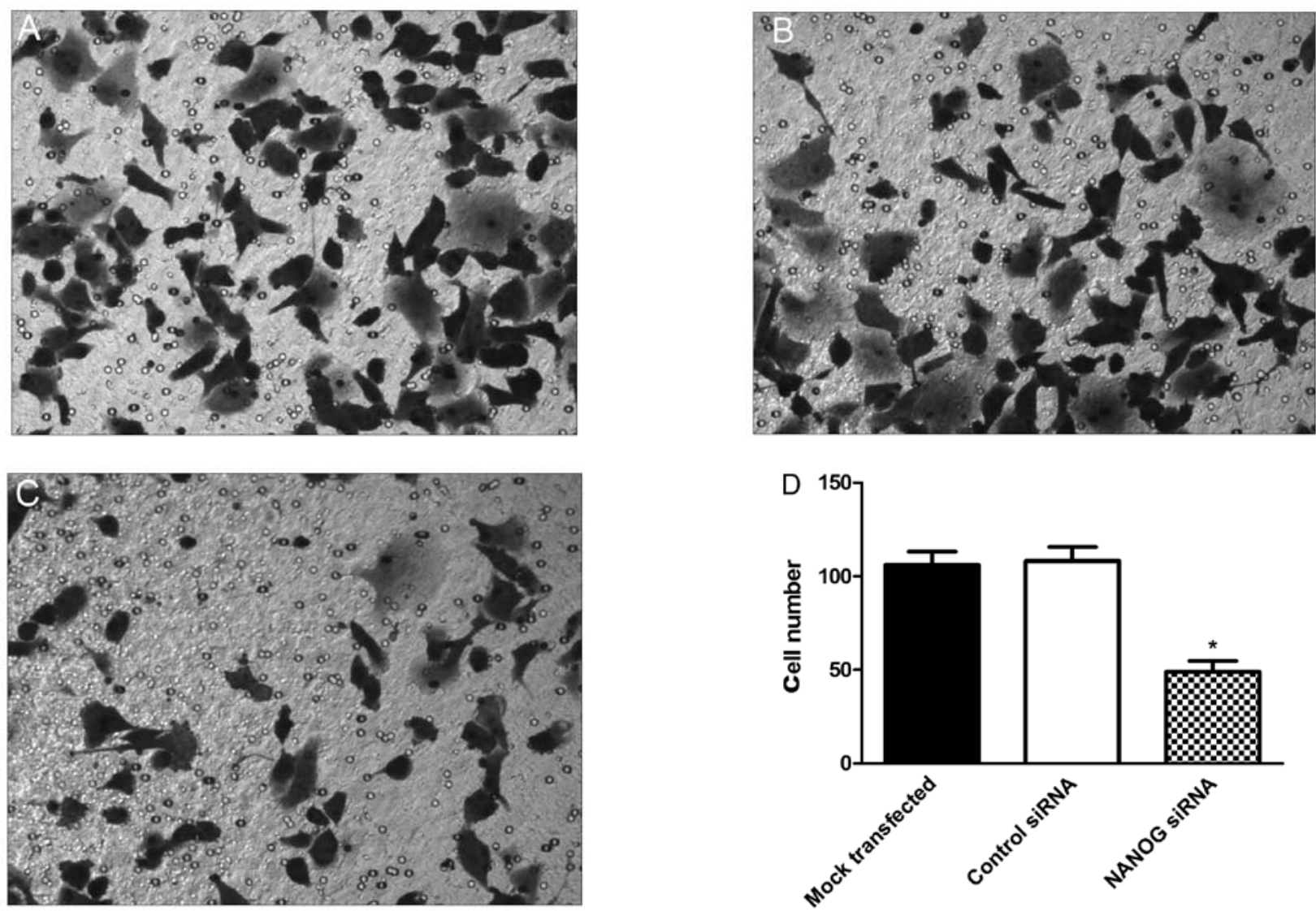

Figure 3. Knockdown of NANOG inhibited cell migrating ability of HepG2 cells. (A-C) The migrating ability of cells with knockdown of NANOG expression was decreased in comparion with the mock transfected and control siRNA tranfected cells; (A) mock transfected HepG2 cells; (B) control siRNA tranfected HepG2 cells; (C) NANOG siRNA tranfected HepG2 cells; and (D) migrating cell number. Values are mean \pm standard deviation. "P<0.05 vs. mock transfected cells and control siRNA transfected cells.
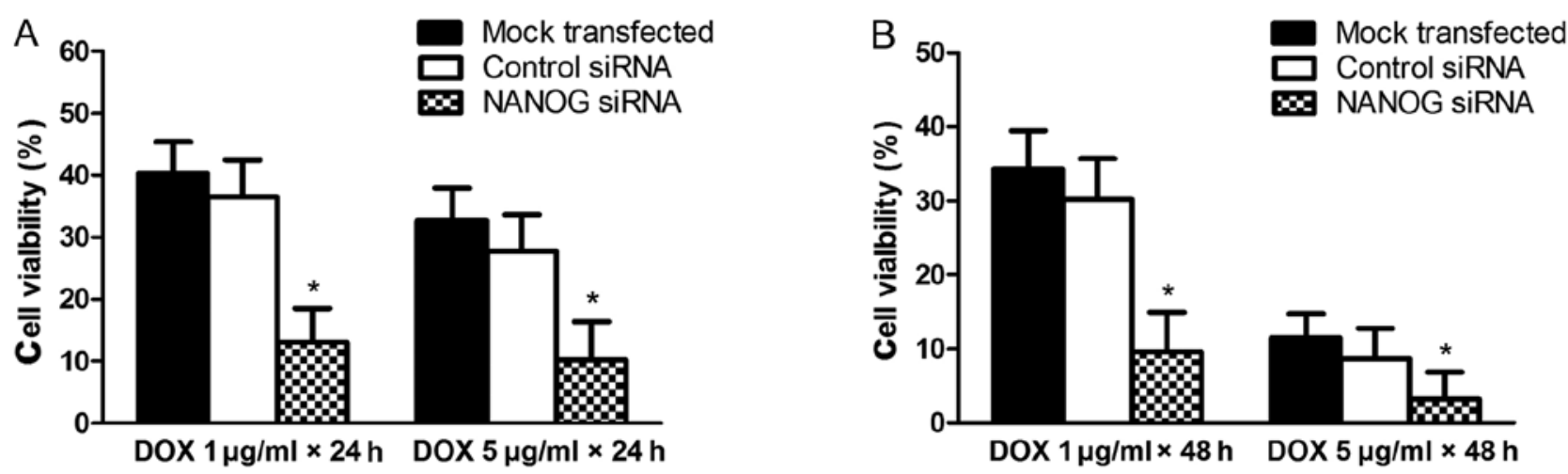

Figure 4. Knockdown of NANOG sensitized cells to doxorubicin. HepG2 cells were transfected with NANOG siRNA or control siRNA for $24 \mathrm{~h}$, then exposed to various concentrations of doxorubicin for 24 or $48 \mathrm{~h}$ and the viability was accessed. (A and B) The cell viability experiments revealed that treatment of HepG2 cells with NANOG-siRNA resulted in more enhanced chemosensitivity to doxorubicin in comparion with the mock transfected and control siRNA tranfected cells; values are mean \pm standard deviation. ${ }^{*} \mathrm{P}<0.05$ vs. mock transfected cells and control siRNA transfected cells.

the expression of MDR1 was significantly decreased in NANOG siRNA transfected cells at both mRNA and protein levels $(\mathrm{P}<0.05)$.

\section{Discussion}

The chemoresistance of cancer cells is one of the important reasons for the failure of liver cancer chemotherapy in clinic.
The cancer stem cells hypothesis may provide a novel idea for the research and treatment of cancer multidrug resistance. The CSC hypothesis posits that cancers contain a small percentage of CSCs possessing the capacity to self-renewal and to cause the heterogeneous lineages of cancer cells $(18,19)$. CSCs are regarded as the cause of tumor formation and recurrence. There is emerging evidence to show the existence of CSCs in various solid cancers including breast cancer, glioma, prostate 

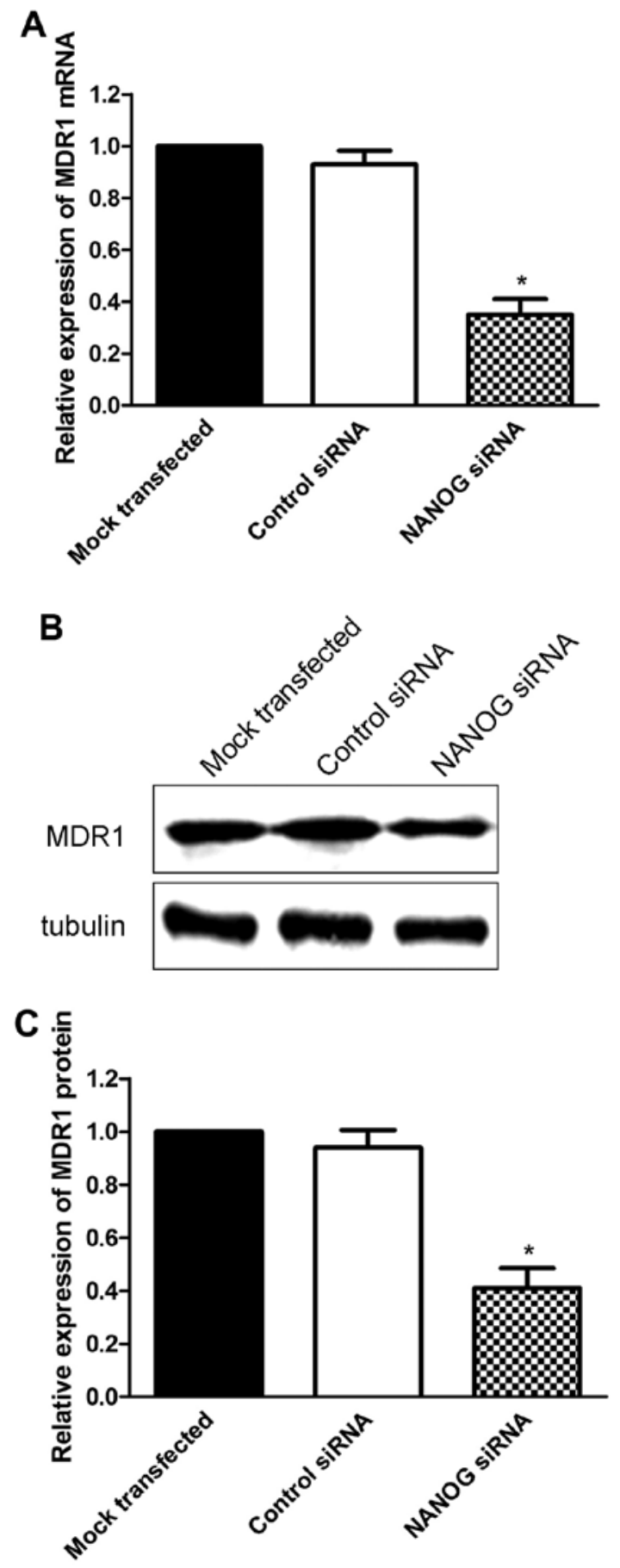

Figure 5. Knockdown of NANOG reduced expression of MDR1 in HepG2 cells. (A) MDR1 mRNA levels assessed by real-time RT-PCR $24 \mathrm{~h}$ after transfection in HepG2cells treated with either NANOG siRNA, or control siRNA, or mock-transfected cells. Data were normalized by using $\beta$-actin as an internal standard. "P $<0.05$ vs. mock transfected cells and control siRNA transfected cells. (B and C) MDR1 protein levels assessed by western blot analysis $48 \mathrm{~h}$ after transfection in HepG2 cells treated with either NANOG siRNA, or control siRNA, or mock transfected cells. Tubulin was used as a loading control. ${ }^{*} \mathrm{P}<0.05$ vs. mock transfected cells and control siRNA transfected cells

cancer $(10,11,20)$, and liver cancer $(21)$. It has been shown that CSCs are resistant to the current chemotherapies (22) and existence of CSCs may be the cause of liver cancer chemotherapy failure. However, the mechanism of why CSCs are resistant is not clearly understood. NANOG is a homeodomain-containing transcription factor that functions to maintain self-renewal and pluripotency of ESCs $(7,8,23)$. Several studies have provided consistent evidence for the role of NANOG as a potential human oncogene $(10,15)$. Aberrant expression of NANOG during tumor development was observed in a variety of different tumor types and cell lines, including HCC (24). In addition, transfection of NANOG cDNA into 293 cells leads to malignant transformation in vitro and tumor formation in vivo (25), and downregulation of NANOG results in decreased long-term clonal and clonogenic growth, reduced proliferation and, in some cases, altered differentiation (15). Moreover, NANOG was overexpressed in CD24 positive HCC cells, which possessed the traits of stem/progenitor cells (26). Using the NANOG promoter as a reporter system, a small subpopulation of NANOG-positive cells isolated from HCC cell lines, exhibited enhanced ability of self-renewal, clonogenicity and initiation of tumors, which are consistent with crucial hallmarks in the definition of CSCs in HCC (27). Furthermore, according to the CSC hypothesis, CSCs are resistant to anticancer agents and the rare population of CSCs can be enriched upon chemotherapy (20). It was reported that a well-established MDR cell line K562/A02 enriched by doxorubicin from K562 cells exhibited tumor-initiating properties, and the expressions of NANOG in K562/A02 cells were elevated in comparison to parental K562 cells, indicating a possible correlation between NANOG expression and doxorubicin resistance (28). These findings indicated that NANOG plays a particularly important role in chemoresistance of liver cancer cells or CSCs.

To test the hypothesis whether NANOG is involved in chemoresistance in HCC, we first used lipofectaminemediated siRNA technology to knock down the expression of NANOG in human liver cancer cell line HepG2. We found that both mRNA and protein levels of NANOG expression were significantly inhibited in the NANOG siRNA transfected HepG 2 cells detected by real-time PCR and western blot assay. The HepG2 cells transfected with control siRNA or with lipofectamine only did not inhibit the expression of NANOG, indicating the effect of siRNA-mediated knockdown of NANOG. Then we examined the effect of NANOG on the biological characteristics of colony formation capacity and cell migration ability of human liver cancer cells. Our data showed the colony formation rate of NANOG siRNA transfected HepG2 cells was lower than the mock transfected and control siRNA transfected cells, and there were less migrating cells in NANOG siRNA transfected HepG 2 cells than in the other cell lines. These results indicated that the knockdown of NANOG expression inhibited the colony formation capacity and cell migration ability of human liver cancer cell line HepG2. The HepG2 cells transfected with or without NANOG siRNA were treated with doxorubicin to evaluate the chemosensitivity of cells. We found that the chemosensitivity to doxorubicin was increased when the NANOG expression levels in HepG2 cells were inhibited, compared to the mock transfected and control siRNA transfected cells. These data indicate that aberrant expression of NANOG in liver cancer cells may be associated with cancer cell resistance to doxorubicin and inhibition of NANOG expression may be a novel potential strategy for sensitizing liver cancer cells to doxorubicin. 
Studies have shown that the failure of chemotherapy in many malignant tumors was partially associated with abnormal expression of MDR1 gene, which encodes the P-glycoprotein to pump anticancer agents out of the cells $(29,30)$. Knockdown of MDR1 gene in malignant cancer cells can restore their sensitivity to anticancer agents $(31,32)$, indicating that MDR1 gene plays an importance role in the multidrug resistance of HCC to doxorubicin. To further verify whether the effect of NANOG in regulating sensitivity to doxorubicin was correlated with MDR1 gene, we examined the expression of MDR1 mRNA and protein in HepG2 cells with or without NANOG knockdown. We found that when the NANOG expression was inhibited by siRNA-mediated silence, the expression of MDR1 at mRNA and protein levels in HepG2 cells was decreased in comparison to parental HepG2 cells without the knockdown of NANOG, indicating that knockdown of NANOG expression downregulates the expression of MDR1 gene in HepG2 cells. These data suggested that NANOG may be correlated with the expression of MDR1 gene and further altered the chemosensitivity of human liver cancer to doxorubicin. Although the underlying mechanism of NANOG in regulating MDR1 gene expression and chemoresistance still remains unclear, these result indicated that aberrant expression of NANOG may be closely related to the malignant characteristics including multidrug resistance of liver cancer and inhibition of NANOG expression may be a new approach for sensitizing liver cancer cells to chemotherapeutic drugs to reverse MDR in HCC patients.

In conclusion, our present data suggested that the knockdown of NANOG expression decreased the colony formation capacity, invasiveness ability and doxorubicin resistance of human liver cancer cell line HepG2. In addition, inhibition of NANOG expression in human HepG2 cells resulted in decreased MDR1 expression and increased chemosensitivity to doxorubicin and NANOG might serve as a novel potential therapeutic target to reverse multidrug resistance of liver cancer.

\section{Acknowledgements}

This study was supported by National Natural Science Foundation of China (nos. 30872485 and 81000889).

\section{References}

1. Okuda K: Hepatocellular carcinoma. J Hepatol 32: 225-237, 2000.

2. Parkin DM, Bray F, Ferlay J and Pisani P: Estimating the world cancer burden: Globocan 2000. Int J Cancer 94: 153-156, 2001.

3. Lee JH, Chung YH, Kim JA, et al: Genetic predisposition of hand-foot skin reaction after sorafenib therapy in patients with hepatocellular carcinoma. Cancer 119: 136-142, 2013.

4. Sauer G, Kafka A, Grundmann R, Kreienberg R, Zeillinger R and Deissler H: Basal expression of the multidrug resistance gene 1 (MDR-1) is associated with the TT genotype at the polymorphic site $\mathrm{C} 3435 \mathrm{~T}$ in mammary and ovarian carcinoma cell lines. Cancer Lett 185: 79-85, 2002.

5. Ling V: Multidrug resistance and P-glycoprotein expression. Ann NY Acad Sci 507: 7-8, 1987.

6. Roninson IB, Chin JE, Choi KG, et al: Isolation of human mdr DNA sequences amplified in multidrug-resistant KB carcinoma cells. Proc Natl Acad Sci USA 83: 4538-4542, 1986.

7. Chambers I, Colby D, Robertson M, Nichols J, Lee S, Tweedie S and Smith A: Functional expression cloning of Nanog, a pluripotency sustaining factor in embryonic stem cells. Cell 113: 643-655, 2003.
8. Mitsui K, Tokuzawa Y, Itoh H, et al: The homeoprotein Nanog is required for maintenance of pluripotency in mouse epiblast and ES cells. Cell 113: 631-642, 2003.

9. Freberg CT, Dahl JA, Timoskainen S and Collas P: Epigenetic reprogramming of OCT4 and NANOG regulatory regions by embryonal carcinoma cell extract. Mol Biol Cell 18: 1543-1553, 2007.

10. Ben-Porath I, Thomson MW, Carey VJ, Ge R, Bell GW, Regev A and Weinberg RA: An embryonic stem cell-like gene expression signature in poorly differentiated aggressive human tumors. Nat Genet 40: 499-507, 2008.

11. Gu G, Yuan J, Wills M and Kasper S: Prostate cancer cells with stem cell characteristics reconstitute the original human tumor in vivo. Cancer Res 67: 4807-4815, 2007.

12. Zbinden M, Duquet A, Lorente-Trigos A, Ngwabyt SN, Borges I and Ruiz i Altaba A: NANOG regulates glioma stem cells and is essential in vivo acting in a cross-functional network with GLI1 and p53. EMBO J 29: 2659-2674, 2010.

13. Seigel GM, Hackam AS, Ganguly A, Mandell LM and GonzalezFernandez F: Human embryonic and neuronal stem cell markers in retinoblastoma. Mol Vis 13: 823-832, 2007.

14. Meng HM, Zheng P, Wang XY, et al: Overexpression of nanog predicts tumor progression and poor prognosis in colorectal cancer. Cancer Biol Ther 9: 295-302, 2010.

15. Jeter CR, Badeaux M, Choy G, et al: Functional evidence that the self-renewal gene NANOG regulates human tumor development. Stem Cells 27: 993-1005, 2009.

16. Jeter CR, Liu B, Liu X, et al: NANOG promotes cancer stem cell characteristics and prostate cancer resistance to androgen deprivation. Oncogene 30: 3833-3845, 2011.

17. Du Y, Shi L, Wang T, Liu Z and Wang Z: Nanog siRNA plus Cisplatin may enhance the sensitivity of chemotherapy in esophageal cancer. J Cancer Res Clin Oncol 138: 1759-1767, 2012.

18. Dalerba P, Cho RW and Clarke MF: Cancer stem cells: models and concepts. Annu Rev Med 58: 267-284, 2007.

19. Dalerba P and Clarke MF: Cancer stem cells and tumor metastasis: first steps into uncharted territory. Cell Stem Cell 1: 241-242, 2007.

20. Yu F, Yao H, Zhu P, et al: let-7 regulates self renewal and tumorigenicity of breast cancer cells. Cell 131: 1109-1123, 2007.

21. Xu XL, Xing BC, Han HB, et al: The properties of tumorinitiating cells from a hepatocellular carcinoma patient's primary and recurrent tumor. Carcinogenesis 31: 167-174, 2010.

22. Al-Hajj M: Cancer stem cells and oncology therapeutics. Curr Opin Oncol 19: 61-64, 2007.

23. Boyer LA, Lee TI, Cole MF, et al: Core transcriptional regulatory circuitry in human embryonic stem cells. Cell 122: 947-956, 2005.

24. Tang Y, Kitisin K, Jogunoori W, et al: Progenitor/stem cells give rise to liver cancer due to aberrant TGF-beta and IL-6 signaling. Proc Natl Acad Sci USA 105: 2445-2450, 2008.

25. Lin YL, Han ZB, Xiong FY, et al: Malignant transformation of 293 cells induced by ectopic expression of human Nanog. Mol Cell Biochem 351: 109-116, 2011.

26. Lee TK, Castilho A, Cheung VC, Tang KH, Ma S and Ng IO: $\mathrm{CD} 24(+)$ liver tumor-initiating cells drive self-renewal and tumor initiation through STAT3-mediated NANOG regulation. Cell Stem Cell 9: 50-63, 2011.

27. Shan J, Shen J, Liu L, et al: Nanog regulates self-renewal of cancer stem cells through the insulin-like growth factor pathway in human hepatocellular carcinoma. Hepatology 56: 1004-1014, 2012.

28. Xin H, Kong Y, Jiang X, et al: Multi-drug-resistant cells enriched from chronic myeloid leukemia cells by Doxorubicin possess tumor-initiating-cell properties. J Pharmacol Sci 122: 299-304, 2013.

29. Pérez-Tomás R: Multidrug resistance: retrospect and prospects in anti-cancer drug treatment. Curr Med Chem 13: 1859-1876, 2006.

30. Goda K, Bacsó Z and Szabó G: Multidrug resistance through the spectacle of P-glycoprotein. Curr Cancer Drug Targets 9: 281-297, 2009.

31. Lage H: MDR1/P-glycoprotein (ABCB1) as target for RNA interference-mediated reversal of multidrug resistance. Curr Drug Targets 7: 813-821, 2006.

32. He Y, Bi Y, Hua Y, et al: Ultrasound microbubble-mediated delivery of the siRNAs targeting MDR1 reduces drug resistance of yolk sac carcinoma L2 cells. J Exp Clin Cancer Res 30: 104, 2011. 